\title{
Quality Control of Composite Materials by Neural Network Analysis of Ultrasonic Power-Spectra
}

Thomsen, Jon Juel

Published in:

Materials Evaluation

Publication date:

1991

Document Version

Publisher's PDF, also known as Version of record

Link back to DTU Orbit

Citation (APA):

Thomsen, J. J. (1991). Quality Control of Composite Materials by Neural Network Analysis of Ultrasonic PowerSpectra. Materials Evaluation, (May), 594-600.

\section{General rights}

Copyright and moral rights for the publications made accessible in the public portal are retained by the authors and/or other copyright owners and it is a condition of accessing publications that users recognise and abide by the legal requirements associated with these rights.

- Users may download and print one copy of any publication from the public portal for the purpose of private study or research.

- You may not further distribute the material or use it for any profit-making activity or commercial gain

- You may freely distribute the URL identifying the publication in the public portal

If you believe that this document breaches copyright please contact us providing details, and we will remove access to the work immediately and investigate your claim. 


\title{
Quality Control of Composite Materials by Neural Network Analysis of Ultrasonic Power Spectra
}

\author{
by J. J. Thomsen* and K. Lund ${ }^{+}$
}

\begin{abstract}
A new concept of spectral analysis of ultrasonic test measurements is presented. An artificial neural network was implemented in software and trained to classify measured ultrasonic power spectra of composite laminates according to fabrication quality. In a laboratory test, the network was able to classify correctly 83 percent of 365 spectra from epoxy lominate plates in which localized areas of delamination, anomalous fiber concentration, and matrix porosity had been artificially induced.
\end{abstract}

Keywords: acousto-ultrasonic testing, bond testing, composite material, neural network, nondestructive evaluation, quality control.

\section{INTRODUCTION}

Composite materials may fail through a variety of damage modes, including matrix cracking, fiber-matrix debonding, matrix porosity, delamination, and fiber breakage. Detection of such faults quickly, reliably, nondestructively, and automatically is a difficult problem of major scientific and industrial interest. Many widely used techniques are based on acoustoultrasonic power spectra. This paper discusses the possibility of drastically increasing the benefit of these techniques through proper postprocessing of data.

Talreja ${ }^{1}$ proposed a technique, based on the analysis of stress-wave factors (SWFs), for quality control of manufactured composite laminates. SWFs were defined through power spectral moments of a stress wave propagating a certain distance in a material specimen. The basic idea implied, first introduced by Vary and Bowles, ${ }^{2,3}$ was that stress-wave characteristics may reflect microstructural properties and that integrated average measures of such properties can be obtained through power spectral density distributions. Talreja proposed a set of five SWFs, based on spectral moments of ascending order, the first one interpreted as the root mean square (RMS) value of the stress wave. In a laboratory test, this first SWF was found to correlate with fabrication quality in that it was, on average, 25 and 35 percent lower for graphite-epoxy laminates with induced matrix porosities and delaminations, respectively, as compared with undamaged specimens. Talreja concluded that SWFs were able to provide indicators of fabrication quality.

Implied in this conclusion is the assumption that the distributions of SWFs of different fabrication qualities do not overlap significantly. In general, this does not hold true. In the example presented in this paper, different fabrication qualities

\footnotetext{
"Dept. of Solid Mechanics, Technical University of Denmark, DK2800, Lyngby, Denmark; 459319 04, ext. 4255; fax 42882421.

tPerceptronics International, 3 avenue Didier Daurat, 31400 Toulouse, France.
}

did, indeed, result in SWF distributions showing highly significant overlaps. Even if the SWFs on the average were different, they were not significantly so, and it was not possible to relate any individual SWF measurement to fabrication quality. This does not necessarily imply that power spectra from materials of different fabrication quality were not themselves separable. In fact, the measured power spectra of a normal and a delaminated composite were easily separable by a human observer.

SWF computation represents one approach to the problem of quantifying the essence of a signal shape. However, other feature extractors are possible as well, most refined probably those that evolve in the brain of a human observer studying the different power spectra of, for example, undamaged and delaminated composite specimens. Such an observer learns rather quickly to detect and recognize signal features that separate normal from nonnormal specimens. The mechanisms involved in such a process may be described as an intuitive and gradual development of working hypotheses, which are either rejected or refined according to the outcome of subsequent classification attempts. This ability to find out what is significant and what is not in a given context (i.e., to develop feature extractors) is unique to the human brain. It cannot be exactly copied. But, as will be demonstrated, it can be partly imitated by the so-called artificial neural networks (ANNs). ANNs, simple software or hardware models of the information-processing "wetware" in living brains, have already exhibited some of the remarkable properties of their live exemplars, including the ability, of particular importance in this context, to solve difficult nonlinear classification problems.

In this present work, we implemented an ANN in software and trained it through examples to classify ultrasonic power spectra from composite plates according to four different damage modes. We thus recognize the correlation of power spectra with damage state and put forward a feature-extraction technique alternative to use of SWFs.

In what follows, we shall first summarize the neural network concept in general and the specific network actually implemented. Next, we shall describe the laboratory tests performed, the training of the network, and its posttraining performance.

\section{THE NEURAL NETWORK CONCEPT}

A live neural network consists of a large number of simple computational units (neuron cells) interconnected in a complicated network. Each unit receives information from many other units through incoming lines (dendrites). Incoming information is filtered through a simple nonlinear element, and the result is broadcast to many other units through 
outgoing lines (axons). Axons and dendrites are connected through switches (synapses), which are either excitatory or inhibitory. The network as a whole interacts with the external environment through specialized input/output neurons, and the global operation of the net can then be described as a mapping from input to output, governed by the sequences-ofstate changes, resulting from the process described above, in individual neurons. The process is inherently parallel: each neuron operates independently of others. Speed, therefore, is not tied to net size. Furthermore, information is not stored locally, as in man-made Von Neumann computers; instead, it is distributed all over the network, in the synaptical connections.

ANNs represent recent efforts to imitate this unique structure in hardware or software. This is accomplished through mathematical modeling of the (hypothesized) essential features of live nets, in the hope that similar structures cause similar functions. Some success has already been attained, in that implemented ANNs have proved trainable, self-organizing, fault-tolerant, and fast, as well as able to solve nonalgorithmic problems, to generalize, and to make associations. (See Rumelhart and McClelland ${ }^{4}$ for an explorative, biologically flavored treatment, Lippmann ${ }^{5}$ for a clear, nonbiological introduction, and Baker and Windsor 6 for a recent application in the field of nondestructive evaluation.)

We now turn to the specific ANN used in this study for classification of ultrasonic power spectra. This is the multilayer perceptron, sketched in Figure 1, which belongs to the class of layered feed-forward nets with supervised learning. It consists of neurons organized in fully interconnected layers. There are $N+1$ layers, numbered $\ell=0, N$, and $n$, neurons in each layer. Only the layers 1 to $N$ contain computing neurons. A computing neuron changes its state $x$ according to received input. If this input exceeds an internal firing threshold $\theta$, the state becomes "high"; otherwise, it is "low." Input to a neuron is defined as the total sum of weighted states from neurons in the layer below, the weights $w$ representing excitatory or inhibitory synaptical connections. Information thus flows layerwise forward through the network, initiated by an external excitation of the (noncomputing) input neurons in layer 0 .
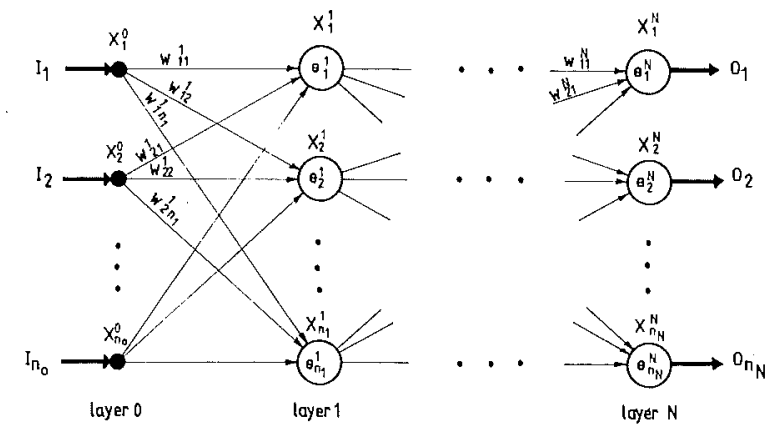

Figure 1-Multilayer perceptron, with neuron states $\mathrm{x}$, synaptical weights $\mathrm{w}$, and firing thresholds $\theta$.

Globally, the computation performed can be thought of as a mapping of $n_{0}$-dimensional input vectors I to $n_{N}$-dimensional output vectors $\mathbf{O}$. Formally, the mapping is given by

(1)

$$
x_{j}^{\rho}=\mathrm{I}_{j}, j=1, n_{0} .
$$

For each layer $\ell=1, N$ and each neuron $j=1, n_{\ell}$

$$
x_{j}^{\zeta}=f\left(\sum_{i=1}^{n_{\ell-1}} w_{i j}^{\ell} x_{i}^{\ell-1}-\theta_{j}^{\ell}\right)
$$

and then

$$
\mathbf{O}_{j}=x_{j}^{N}, j=1, n_{N}
$$

where all variables are real-valued and where $\mathrm{I}_{j}, \mathrm{O}_{j}$ are the $j$ th components of input and output vectors $\mathrm{I} \in R^{n_{0}}, \mathrm{O} \in R^{n_{N}} ; x_{j}^{\ell}$ is the state of the $j$ th neuron in layer $\ell ; w_{i j}^{\ell}$ is the synaptical weight between the $i$ th neuron in layer $\ell-1$ and the $j$ th neuron in layer $\ell ; \theta_{j}^{\ell}$ is the internal firing threshold of the $j$ th neuron in layer $\ell$; and $f$ is the threshold function performed by every neuron.

For any interesting computation to be done, the function $f$ must be nonlinear. Step or ramp functions will do, but present network-training algorithms require that $f$ is differentiable and nondecreasing, as, for example, the frequently used sigmoidal squashing function:

$$
f(\alpha)=\frac{1}{1+e^{-\alpha}} \quad \alpha \in R, f(\alpha) \in[0 ; 1] .
$$

Useful net computation is done when inputs I and outputs $\mathbf{O}$ represent meaningful quantities and when the weights $w$ and thresholds $\theta$ are properly tuned to the type of problem to be solved. For example, I could represent plain text to be transcribed into phonemes, or a desired target position of a robot manipulator arm, or pixel values of an obscured scenery picture. Correspondingly, $\mathbf{O}$ could then represent phonemical codes for a speech machine, or robot motor commands, or labels characterizing a recognized object. In the present work, I represents spectral information on ultrasonic measurements from composite laminates, and $\mathrm{O}$ labels the class of fabrication quality to which the tested specimen is supposed to belong.

In all cases, the desired $\mathrm{I} \rightarrow \mathrm{O}$ mapping must be achieved through proper assignment of synaptical weights $w$ and neuron thresholds $\theta$. This is obtained through network training. During a training phase, representative examples of correct $\mathrm{I} \rightarrow \mathbf{O}$ mappings are repeatedly presented to the network. Each presentation is followed by small adjustments of weights and thresholds if the computed output is not correct.

If there is any systematical relationship between input and output and the training examples are representative of this, and if the network topology is properly chosen, then the trained network will often be able to generalize beyond learned examples (i.e., to give reasonable answers to new questions) and to give correct answers even if inputs are corrupted by noise.

Multilayer network training represents a nonlinear parameter estimation problem in the $(w, \theta)$ parameter space. Hidden neuron layers (between input and output layers) make this problem difficult to solve, so difficult, in fact, that, although a single-layer training algorithm was known in 1959, a multilayer algorithm was first published in 1986, by Rumelhardt et al. ${ }^{7}$ (and independently by others). This so-called back-propagation algorithm employs a gradient-descent search technique for minimizing a cost function, defined as the mean-square difference between desired and actual network outputs. In our notation, the training algorithm may be summarized as follows.

(a) Initialize weights and thresholds: Assign small random numbers to $\theta_{j}^{\ell}$ and $w_{i j}^{\ell}$, for $\ell=1, N, j=1, n_{\ell,} \mathrm{i}=1, n_{\ell-1}$. Zero the iteration counter $t$.

(b) Present an I $\rightarrow$ O example: Pick at random from the set of training examples an input vector $\mathbf{I}_{j}, j=1, n_{0}$, and the associated correct output vector $\overline{\mathrm{O}}_{j}, j=1, n_{N}$. If the net is to be used as a classifier and the actual input belongs to class $q$, then $\overline{\mathbf{O}}_{j}=0.9$ for $j=q$, whereas $\overline{\mathbf{O}}_{j}=0.1$ for $j \neq q$. (The numbers 0.9 and 0.1 represent "high" and "low" neuron states.) Advance the iteration counter $t$ by one.

(c) Compute actual outputs: Use Equations 1-3 to compute the outputs $\mathbf{O}_{j}, j=1, n_{N}$ given inputs $\mathbf{I}_{j}$ and instantaneous values of $w$ and $\theta$.

(d) Adjust weights $w$ and thresholds $\theta$ : Start with output neurons, and work backward through the net. For $\ell=$ $N, N-1, \ldots, 1$; for $j=1, n_{\ell}$ for $i=1, n_{\ell-1}$ : 
(5)

where

(6)

$$
w_{i j}^{\ell}(\mathrm{t}+1)=w_{i j}^{\ell}(t)+\Delta w_{i j}^{\ell}(t)
$$

$$
\Delta w_{i j}^{\ell}(t)=\eta \delta_{j}^{\complement} x_{i}^{\ell-1}
$$

gives the weight corrections. Here $\eta$ is the learning gain, to be chosen as large as possible without leading to intolerable oscillations (typically $0.01-0.9$ ), and $\delta_{j}^{f}$ is the error term, which, for sigmoidal nonlinearities (Equation 4), is given by

$$
\delta_{j}^{\prime}= \begin{cases}O_{j}\left(1-\mathbf{O}_{j}\right)\left(\bar{O}_{j}-O_{j}\right) & \text { for } \ell=N \\ x_{j}^{\ell}\left(1-x_{j}^{\ell}\right) \sum_{k=1}^{n_{\ell+1}} \delta_{k}^{\ell+1} w_{j k}^{\ell+1} & \text { for } \ell=N-1, \\ & N-2, \ldots, 1 .\end{cases}
$$

(The first two terms of both equations represent the derivative of the threshold nonlinearity. For the sigmoid Equation 4 used here, $f^{\prime}(\alpha)=f(\alpha)[1-f(\alpha)]$. For other functions, these terms must be replaced accordingly.)

Firing thresholds $\theta$ can be adjusted in the same way as weights by treating them as weights on virtual synaptical connections with constant inputs. This can be seen by rewriting the total input $\tilde{x}$ to a neuron (see Equation 2):

$$
\begin{aligned}
\tilde{x}_{j}^{f} & =\sum_{i=1} w_{i j}^{\ell-1} x_{i}^{\ell-1}-\theta_{j}^{\ell} \\
& \mathrm{n}_{\ell-1} \sum_{i=0}^{\ell} \mathrm{w}_{i j}^{\ell} x_{i}^{\ell-1} \text { for } w_{0 j}^{\ell} \equiv-\theta_{j}^{\ell} \text { and } x_{0}^{\zeta-1}=1 .
\end{aligned}
$$

To stimulate learning convergence, a momentum term that filters out high-frequency fluctuations can be added, so that Equation 5 is replaced by

$$
\Delta w_{i j}^{\ell}(\mathrm{t})=\eta \delta_{j}^{\zeta} x_{i}^{\ell-1}+\alpha \Delta w_{i j}^{\ell}(t-1)
$$

where $\alpha \in[0 ; 1[$ quantifies the influence of preceding weight changes. In suppressing oscillations, the momentum term allows a higher gain $\eta$.

(e) Repeat from point (b) or stop when weights no longer change and the process has converged.

When the training has been completed, the network is ready for use.

Because the parameter estimation problem is nonlinear in $w$ and $\theta$, convergence cannot be proved a priori, and the process may get stuck in a local minimum. In practice, this rarely occurs when the network architecture is properly chosen and consistent sets of training examples are employed. Some inconsistency in training data may be tolerated, but correct $\mathrm{I} \rightarrow \mathbf{O}$ examples must be in the majority. Too few layers of neurons, or too few neurons in each layer, will impede or make impossible any convergence. Too many neurons will generate numerous local minima of identical (minimum) cost. If convergence occurs in such a net, it will often demonstrate a kind of "root learning," in which learned examples are correctly dealt with but generalization to new data is poor. If the net is to be used as an associative memory, this is no problem. If, however, generalizing capabilities are important, the number of net "unknowns" should not greatly exceed the number of net "equations"; i.e., in general,

$$
\sum_{\ell=1}^{N}\left(n_{\ell-1}+1\right) n_{\ell}<n_{N} \cdot M
$$

where $M$ is the number of training examples.

The number of neuron layers should be chosen according to the complexity of the problem. Problem complexity is to be associated with the shape of decision regions necessary to discriminate between different inputs. That is, inputs are supposed to belong to a finite number of classes. The task of a network is to classify inputs according to given examples. To train a network is equivalent to forming those decision regions in the $n_{0}$-dimensional input space that separate training examples belonging to different classes from each other. To a network, a problem is thus complicated if it requires the formation of complex decision region boundaries.

The possible complexity of decision boundaries is determined by the number of network layers. A one-layer network $(N=1)$ is able to form hyperplanes in input space. It can classify inputs only if classes are linearly separable, such as all basic Boolean operations except the XOR (and its negation). A two-layer net $(N=2)$ can form any convex (open or closed) region in hyperspace, such as required by the XOR problem. A three-layer net $(N=3)$ is then capable of forming arbitrarily complex decision regions, limited only by the number of neurons in each layer, and to separate meshed class regions. Thus, more than three computing layers should not be required for any application.

The basic idea underlying the present study is that if ultrasonic power spectra of composite materials are by any means indicative of fabrication quality, they will be clustered in classes that could prove separable to a multilayer perceptron. To test that hypothesis, the authors implemented a multilayer network in software and measured its performance under laboratory conditions, as described below.

\section{AN EXAMPLE}

The neural network approach was tested in a laboratory environment in a specific case: the assessment of fabrication quality of glass-epoxy laminate plates. In the following, we first describe the test specimens, the measurement setup, and the measurements, together with the results of an SWF analysis. We then describe the results obtained using a multilayer ANN.

\section{Test Specimens}

Four glass-epoxy laminate plates (dimensions 550 by 118 by $10 \mathrm{~mm}$, fiber direction longitudinal) were prepared by Renault SA France as follows: plate $r-$ reference plate, i.e., normal curing in autoclave at recommended temperature and pressure; plate $\mathrm{d}-$ delaminated in well-defined zones through insertions of chopped pieces of Teflon ${ }^{\mathrm{TS}}$ prior to curing; plate $\mathrm{f}$-fiber concentration higher in the longitudinal middle third than in the rest of the plate, which was as plate $r$; and plate $\mathrm{p}$-porous matrix material, due to curing without vacuum. The type of flaw associated with each plate is denoted by capital letters: R (reference), D (delaminated), F (fiber concentration), and $P$ (porous). Plate $r$ is thus of status $R$, plate $d$ of status $R / D$, plate $f$ of status $R / F$, and plate $p$ of status $P$.

\section{Measurement Sefup}

Two ultrasonic transducers were placed on the upper face of a test specimen, one sending a pulse and the other receiving the transmitted stress wave. The transducers were held in a fixture that kept their centers $43 \mathrm{~mm}$ apart and were coupled to the specimen surface through a coupling fluid. Both transducers were broadband $(10 \mathrm{MHz})$, with a resonant frequency of $1 \mathrm{MHz}$ and a diameter of $12 \mathrm{~mm}$. The transducer fixture was fixed in space, but the specimens were fixed on a movable-coordinate table, allowing accurate determination of transducer positions.

The received stress-wave signal was amplified and passed to a signal analyzer, in which it was digitized and Fouriertransformed to yield the power spectral density distribution of the stress wave. To facilitate further analysis, each spectrum, originally containing 1024 lines, was normalized by its mean power and lumped into 40 bands in the range 0.2-1.2 MHz. The normalized spectrum and its mean power were then stored, together with specimen label and measurement location, as one measurement result. 


\section{Measurements}

On each of the four specimens $r, d, f$, and $p$, ultrasonic measurements were carried out pointwise along a rectangular grid, spacing $15 \mathrm{~mm}$ longitudinally and $10 \mathrm{~mm}$ transversely. A measurement "point" was (somewhat arbitrarily) defined as the center of a line joining the two transducer centers. In all measurements, the transducers were aligned across the longitudinal fiber direction. Transversely, the outer edges of each measurement grid were aligned as close as possible to the edges of the specimen so as to include measurements affected by boundary effects. Lengthwise, the grid span was chosen to cover representative regions of flawed and nonflawed material. The length span thus differed between specimens.

Prior to the measurements, all grid points were assigned a flaw code $(R, D, F, P)$, easily judged by visual inspection of the transparent material. After each measurement, the power spectrum associated with a grid point was stored, together with specimen label ( $r, d, f, p)$, grid position, and visually judged flaw type.

Typical lumped spectra are shown in Figure 2, and the total number of spectra of each specimen and flaw type is illustrated in Table 1.

TABLE 1 Number of Measured Power Spectra of Specimens ${ }^{a}$

\begin{tabular}{cccccr}
\hline & R & D & F & P & Total \\
\hline $\mathrm{r}$ & 95 & - & - & - & 95 \\
$\mathrm{~d}$ & 28 & 161 & - & - & 189 \\
$\mathrm{f}$ & 40 & - & 48 & - & 88 \\
$\mathrm{p}$ & - & - & - & 48 & 48 \\
\hline Total & 163 & 161 & 48 & 48 & 420
\end{tabular}

aSpecimens $r, d, f$, and $p$, defective through flaws of types $R, D, F$, and $P ; r / R$, reference; $d / D$, delaminated; $f / F$, fiber concentration; $p / P$, porous. Four obviously faulty spectra were excluded from the set.
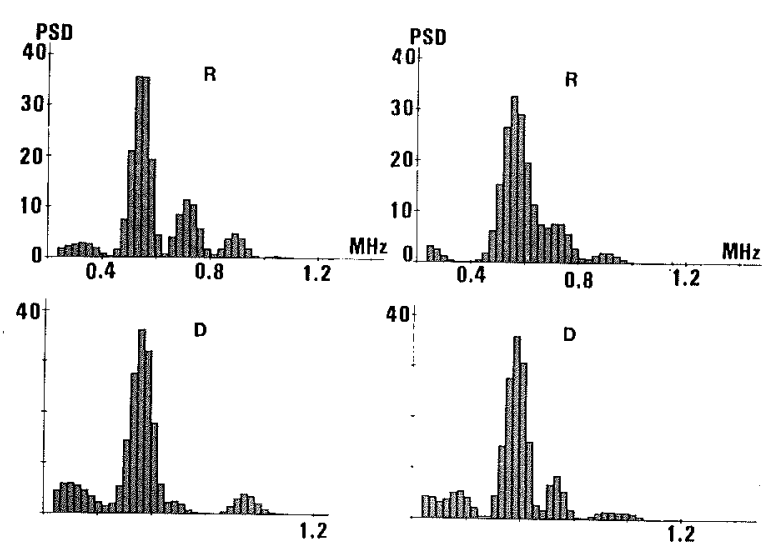

40
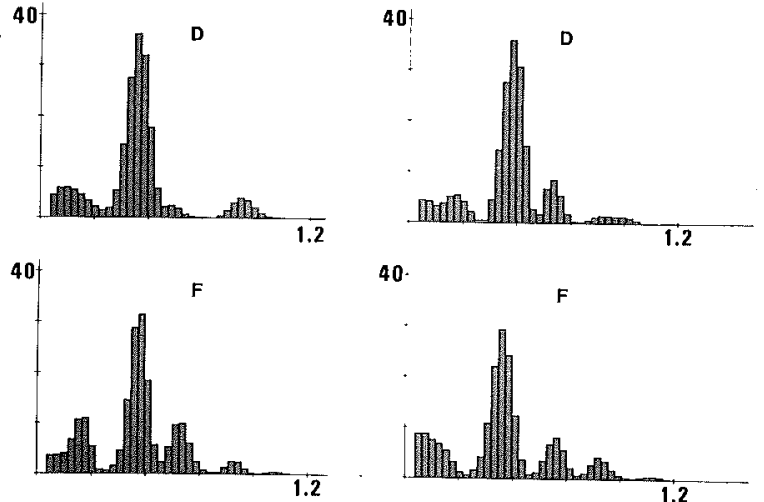

40
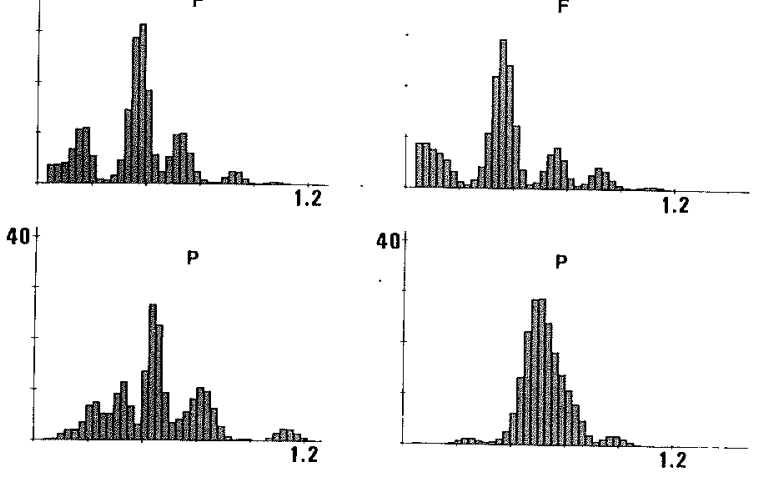

Figure 2-Typical ultrasonic power spectral densities of glass-epoxy plates subject to different flaws. $R$, normal reference; $D$, delaminated; $F$, high fiber concentration; $P$, porous matrix. All spectra are normalized by mean power.

\section{Stress-Wave Factor Analysis}

SWFs were defined through statistical moments $M_{n}$ of measured power spectral density distributions $S(f)$ :

$$
M_{n}=\int_{f_{1}}^{f_{2}} f^{n} S(f) d f
$$

where $n$ represents the order of $M, f$ is frequency, and $f_{1}$ and $f_{2}$ are the lower and upper cutoff frequencies.

Using the discrete equivalent of Equation 11, we computed, for each of our (unnormalized) ultrasonic measurements, the set of five SWFs defined by Talreja ${ }^{1}$ : $S W F_{1}=M_{0}$ (area of power spectrum), $\mathrm{SWF}_{2}=\mathrm{M}_{1} / M_{0}$ (centroid frequency), $\mathrm{SWF}_{3}=\sqrt{M_{2} / M_{0}}$ (mean frequency of mean upcrossings), $\mathrm{SWF}_{4}=\sqrt{M_{4} / M_{2}}$ (frequency of peaks), and $\mathrm{SWF}_{5}=$ $M_{3} M_{0} / M_{2} M_{1}$ (skewness). SWFs were computed for all of the 420 measured spectra quantified in Table 1. In Figure 3, we show the empirical distributions of $\mathrm{SWF}_{1}$, which should be especially sensitive to flaws influencing stress-wave transmission.

From Figure 3, it appears that $\mathrm{SWF}_{1}$ was significantly lowered by porosity $(\mathrm{P})$ flaws. It appears also that distributions associated with the three other types of flaws are heavily overlapping. Although $\mathrm{SWF}_{1}$ on average is different for R-, D-, and F-flaws, it is not significantly so. In fact, in this specific case, a single $\mathrm{SWF}_{1}$ measurement will not allow any reliable estimate of fabrication quality, except for P-flaws.

Similar distributions of factors of higher order $\left(\mathrm{SWF}_{2-5}\right)$ appeared to overlap to a degree at which not even P-flaws could be separated. Nor did plotting the computed SWF values on the measurement grids reveal any indication that any of the SWFs should correlate with flaw regions other than P. We also examined the possibility that distributions would prove separable when viewed as truly multivariate, i.e., in the space spanned by all five SWFs. This was not the case. In conclusion, in this specific case, SWFs were inadequate as indicators of fabrication quality.

Now, this conclusion does not necessarily remain valid under different conditions. Other transducer arrangements, especially the orientation with respect to specimen fibers, and/or additional signal conditioning prior to SWF computation, might result in more-productive conclusions. However, we did not try alternative measurement procedures to improve the indicative power of the SWFs. Instead, we performed a neural network analysis of the same set of measured spectra for which the SWF analysis failed. This is described below.

\section{Neural Network Analysis}

\section{Network Software}

The software consisted of three parts: (1) a preprocessing program, which prepared, checked, and visualized inputs to the network, (2) a flexible general-purpose and graphically supported neural network-simulating program, which allowed easy experimentation with network architectures and parameters, and (3) a postprocessing program, which performed analysis and display of network output, together with automized reporting of all relevant analysis data.

All software was developed by the authors and implemented on a medium-sized workstation (Apollo DN-3500).

\section{Network Architecture}

A typical network used for glass-epoxy flaw detection is shown in Figure 4. It has 21 input neurons, corresponding to 20 components of a normalized measured-power spectral density function and to the constant of normalization. Input spectra were identical to those used for SWF analysis, except for a normalization by mean power and a lumping into 20 frequency bands.

The four output neurons label the class to which the instantaneous input spectrum is supposed to belong: $R$ (normal reference), D (delaminated), F (abnormal fiber concentra- 

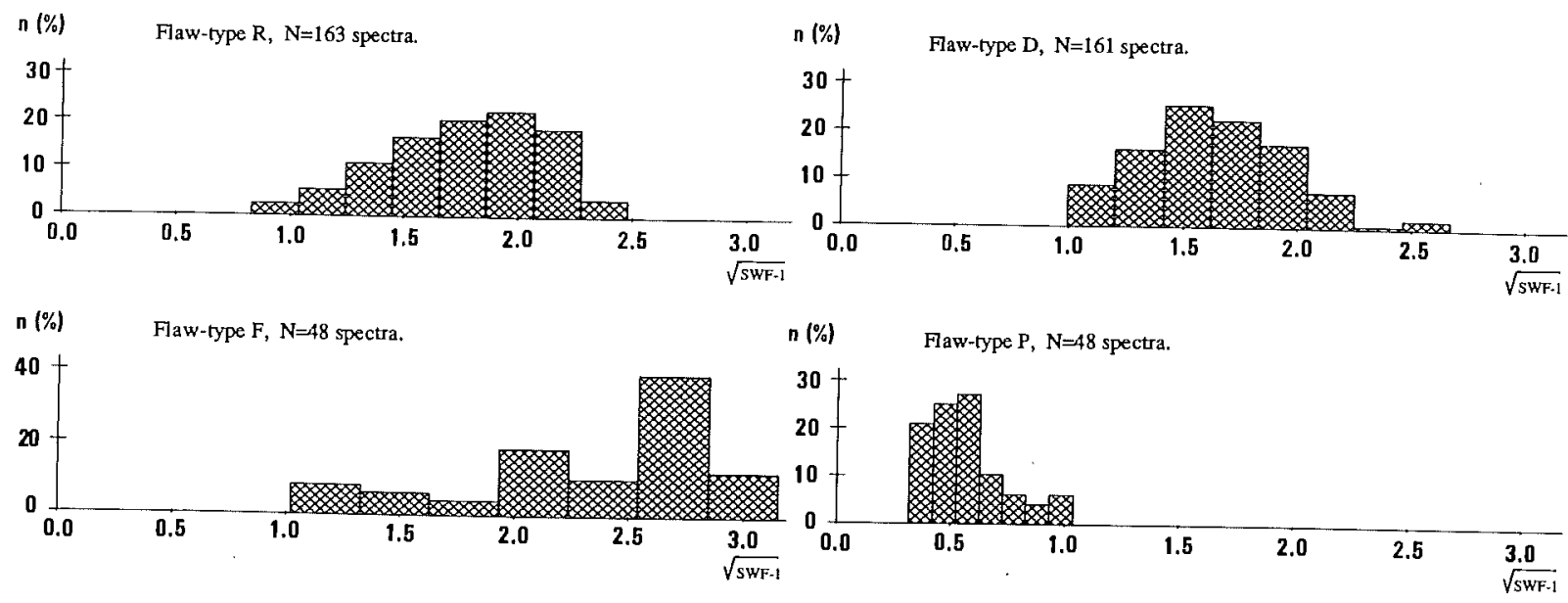

Figure 3-Empirical distributions of $S W F_{1}$ of ultrasonic power spectra from glass-epoxy plates subject to flazus $R, D, F$, and $P$.

tion), or $\mathrm{P}$ (porous matrix). If there is no doubt as to which class a given spectrum belongs, the corresponding output neuron will respond with a "high" output $(\sim 0.9)$; the others will be "low" $(\sim 0.1)$.

The number of hidden layers between input and output, as well as the number of neurons in each of these layers, was subject to experimentation. Systematic trials showed that the choice was not critical as long as at least one hidden layer with at least four neurons was present. The results described below were obtained with one hidden layer of five neurons, as in Figure 4 .

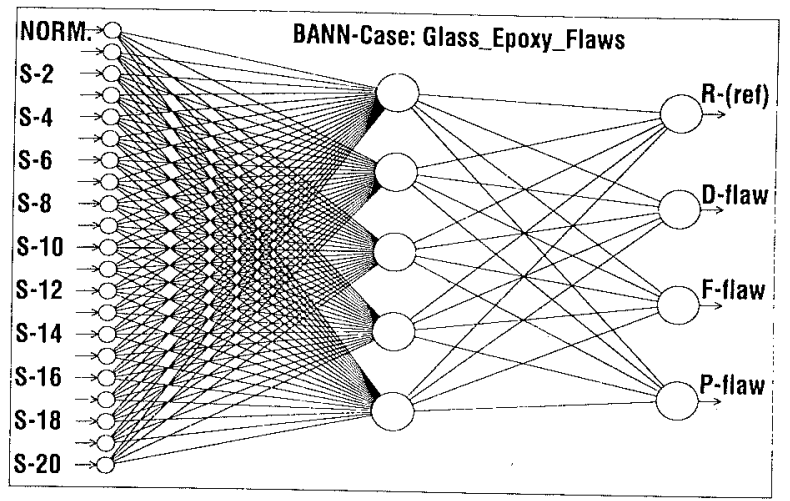

Figure 4-Sample architecture of the neural networks used for flaw detection in glass-epoxy plates.

\section{Network Training}

Some of the 420 measured spectra quantified in Table 1 were extracted for network training.

For each of the four glass-epoxy plates, all measurements from within a rectangular minor section of the measurement grid were selected as training examples. Training areas were chosen so as to cover regions subject to the typical flaw associated with each plate, but otherwise arbitrarily. The training set is quantified in Table 2. Note that only one type of flaw is represented in the training examples from each plate, so that, for example, R-spectra were trained only from plate $r$, although present in plates $\mathrm{d}$ and $\mathrm{f}$ as well.
TABLE 2 Measured Power Spectra and Spectra Extracted for Neural Network Training

\begin{tabular}{ccccc}
\hline Plate & $\begin{array}{c}\text { Total No. } \\
\text { of Spectra }\end{array}$ & $\begin{array}{c}\text { Flaw } \\
\text { Type }\end{array}$ & $\begin{array}{c}\text { No. of } \\
\text { Training Spectra }\end{array}$ & $\begin{array}{c}\text { Flaw } \\
\text { Type }\end{array}$ \\
\hline $\mathrm{r}$ & 95 & $\mathrm{R}$ & 24 & $\mathrm{R}$ \\
$\mathrm{d}$ & 189 & $\mathrm{R}, \mathrm{D}$ & 15 & $\mathrm{D}$ \\
$\mathrm{f}$ & 88 & $\mathrm{R}, \mathrm{F}$ & 8 & $\mathrm{~F}$ \\
$\mathrm{p}$ & 48 & $\mathrm{P}$ & 8 & $\mathrm{P}$ \\
\hline
\end{tabular}

\begin{tabular}{|c|c|c|c|}
\hline Total & 420 & $\mathrm{R}, \mathrm{D}, \mathrm{F}, \mathrm{P}$ & 55 \\
\hline
\end{tabular}

aFour glass-epoxy plates. (The total set of spectra is elaborated in Table 1.)

Network training was performed using the back-propagation algorithm given earlier. Parameters of the algorithm were chosen as follows (none of them critical): learning gain $\eta=0.07$, momentum coefficient $\alpha=0.3$, initial weights and thresholds uniformly random between \pm 0.03 , random order of example presentations.

After 200 presentations of the 55 training examples $(\sim 2 \mathrm{~min}$ of computer time) the network was trained; i.e., weights did not change and the RMS error of the network stabilized at a low value. In the trained state, the network was able to classify all training examples correctly; that is, when a learned spectrum was presented, only the output corresponding to the correct class took on a "high" $(\sim 0.9)$ value. It should be noted that correct net responses to learned examples are neither a necessary nor a sufficient condition for good performance on untrained data. However, the training result confirms that the learned spectra are in fact separable in hyperspace, although not linearly so, as at least one hidden network layer was required.

\section{Network Performance on Untrained Data}

If the network's correct performance on trained examples is not a consequence of root learning, but of a successful determination of internal feature extractors and decision regions, this will show up by the ability of the network to generalize beyond learned examples to new spectra that have certain features in common with those already learned. To test this ability, those spectra of the total set that were not used for training were presented to the network. Flaw types indicated by the highest responding output neuron were then correlated to flaw types determined (beforehand) by visual inspection.

We first present the results obtained when only R-, D-, and P-flaws were trained, i.e., with no F-flaws in the training set. In this case, all training examples were learned perfectly. The network performance on untrained spectra is summarized in Table 3 and visualized in Figure 5. 
TABLE 3 Neural Network Classification of Untrained Power Spectra from Glass-Epoxy Platesa

\begin{tabular}{|c|c|c|c|c|c|c|}
\hline \multirow[b]{2}{*}{ Plate } & \multirow{2}{*}{$\begin{array}{l}\text { No. of } \\
\text { Untrained } \\
\text { Spectra }\end{array}$} & \multicolumn{4}{|c|}{$\begin{array}{l}\text { No. of Correctly Classified } \\
\text { Untrained Spectra }\end{array}$} & \multirow{2}{*}{$\begin{array}{c}\text { No. of } \\
\text { Misclassified } \\
\text { Spectra }\end{array}$} \\
\hline & & $\mathbf{R}$ & $\mathrm{D}$ & $\mathrm{F}$ & $\mathrm{P}$ & \\
\hline $\mathrm{r}$ & 71 & $\begin{array}{c}68 \\
(96 \%)\end{array}$ & - & - & - & $3 \mathrm{R} \rightarrow \mathrm{D}$ \\
\hline d & 174 & $\begin{array}{c}25 \\
(89 \%)\end{array}$ & $\begin{array}{c}133 \\
(91 \%)\end{array}$ & - & - & $13 \mathrm{D} \rightarrow \mathrm{R}, 3 \mathrm{R} \rightarrow \mathrm{D}$ \\
\hline $\mathrm{f}$ & 88 & $\begin{array}{c}32 \\
(80 \%)\end{array}$ & - & 0 & - & $\begin{array}{l}5 \mathrm{R} \rightarrow \mathrm{D}, 3 \mathrm{R} \rightarrow \mathrm{P}, \\
32 \mathrm{~F} \rightarrow \mathrm{R}, 14 \mathrm{~F} \rightarrow \mathrm{D}, \\
1 \mathrm{~F} \rightarrow \mathrm{P}, 1 \mathrm{~F} \rightarrow ?\end{array}$ \\
\hline $\mathrm{p}$ & 40 & - & - & - & $\begin{array}{c}36 \\
(90 \%)\end{array}$ & $4 \mathrm{P} \rightarrow \mathrm{D}$ \\
\hline Total & 373 & $\begin{array}{c}125 \\
(90 \%)\end{array}$ & $\begin{array}{c}133 \\
(91 \%)\end{array}$ & 0 & $\begin{array}{c}36 \\
(90 \%)\end{array}$ & 79 \\
\hline
\end{tabular}

aplates $r, d, F$, and $p$, subject to flaws of type $R, D, F$, and $P$. The network was trained with 47 spectra representing all flaw types except $F$. The percentages given are match scores, referring to the actual occurrence of flawed plate points of specified type.

As will be seen from Table 3, the network performed very well on untrained spectra, except for the F-flawed spectra, which the net had never seen before. Approximately 90 percent of all spectra originating from measurement points of types R, D, or P were classified correctly. Of the 79 misclassified spectra, 48 represented the total set of unknown F-flaws; another 24 represented $R \leftrightarrow D$ confusions. The latter are mainly explained by the low spatial resolution of the measurement arrangement, the two ultrasonic transducers always spanning five grid points, which will blur any appearance of sharp boundaries between two zones of different flaw type.

Figure 5 further visualizes the performance of the network and sheds some light on the resolution problem. For R-, D-,

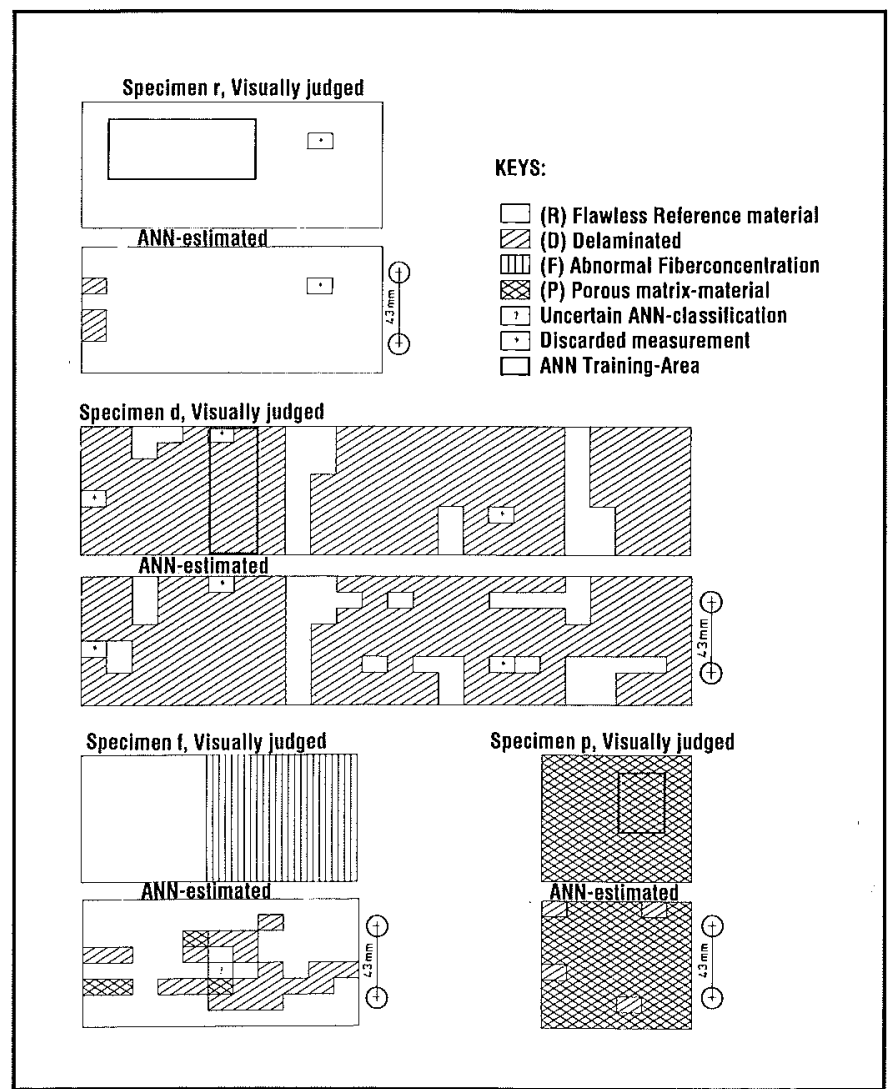

Figure 5-Visually judged and neural network estimates of fabrication quality of four glass-epoxy plates. Network-trained to recognize $R_{-}, D_{-}$, and P-flaws only (training areas marked). Measurement points are represented by small boxes, shaded according to flaw type. Transducers are shown at the far right, roughly to scale. and P-flaws, the neural network flaw estimates differ from the visually judged only at flaw boundaries and at a few isolated points. Considering the dimensions of the transducer arrangement, the resolution was not bad. Note that the network was able to identify the unflawed $(R)$ points of plate $d$, although $R$-points were never trained for that plate.

Naturally, the network could not classify the F-flawed part of plate $f$, as no F-flaws were present in the training set. Therefore, spectra are classified according to the network's limited experience of R-, D-, and P-flaws only, leaving a somewhat unclear picture, although it is clear that a significant portion of the right half of plate $f$ is not normal.

Including eight F-spectra in the training set, and repeating the whole analysis, the network performed as summarized in Table 4 and visualized in Figure 6 for plate $\mathrm{f}$. As will be seen from Figure 6, the network now recognizes the F-flawed half of plate $f$, although the unflawed left half is still not fully accepted as such. From Table 4, it appears that F-flawed spectra in the training set affect the classification of unflawed points of plate $\mathrm{d}$ in that some R-regions are classified as F-flawed. These regions cover 15 points along the edges of the plate, indicating that boundary effects are blurring the very small differences between R- and F-spectra. This similarity between R-and F-spectra, which also appears from Figure 2, is explained by the measurement configuration: in all our measurements, the ultrasonic transducers were aligned across the fiber direction, whereas optimal sensitivity to variations in fiber concentration is to be expected along the fiber direction.

The performance of any flaw-detection method will depend heavily on the quality of the measured data. In the example presented above, the quality of data precluded any productive outcome of an SWF analysis. In contrast, the proposed neural network approach allowed an almost correct estimation of flawed regions. This suggests that more information can be
Specimen $f$, Visually judged

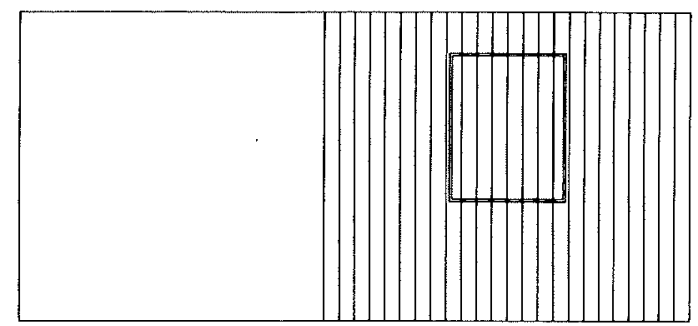

ANN-estimated

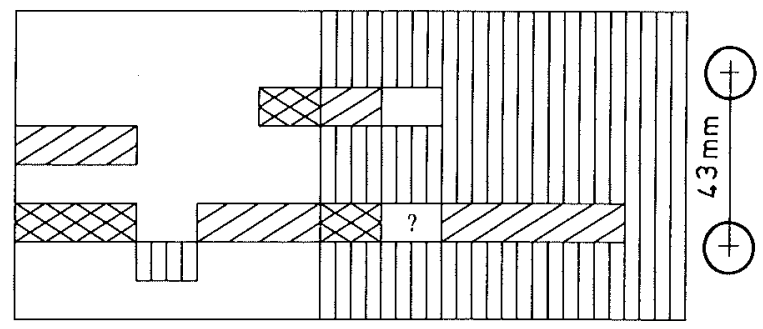

Figure 6-Visually judged and neural network estimates of fabrication quality of plate $f$, network-trained to recognize flaw types $R, D$, $F$, and $P$. See Figure 5 for shading key. 
extracted from ultrasonic power spectra than is possible through SWF analysis.

TABLE 4 Neural Network Classification of Untrained Power Spectra from Glass-Epoxy Plates ${ }^{a}$

\begin{tabular}{|c|c|c|c|c|c|c|}
\hline \multirow[b]{2}{*}{ Plate } & \multirow{2}{*}{$\begin{array}{l}\text { No. of } \\
\text { Untrained } \\
\text { Spectra }\end{array}$} & \multicolumn{4}{|c|}{$\begin{array}{l}\text { No. of Correctly Classified } \\
\text { Untrained Spectra }\end{array}$} & \multirow{2}{*}{$\begin{array}{l}\text { No. of Mis- } \\
\text { classified } \\
\text { Spectra }\end{array}$} \\
\hline & & $\mathrm{R}$ & D & $\mathrm{F}$ & $\mathrm{P}$ & \\
\hline $\mathrm{r}$ & 71 & $\begin{array}{c}70 \\
(99 \%)\end{array}$ & - & - & - & $1 \mathrm{R} \rightarrow \mathrm{D}$ \\
\hline $\mathrm{d}$ & 174 & $\begin{array}{c}11 \\
(39 \%)\end{array}$ & $\begin{array}{c}125 \\
(86 \%)\end{array}$ & - & - & $\begin{array}{l}2 \mathrm{R} \rightarrow \mathrm{D}, 15 \mathrm{R} \rightarrow \mathrm{F}, \\
14 \mathrm{D} \rightarrow \mathrm{R}, 3 \mathrm{D} \rightarrow \mathrm{F},\end{array}$ \\
\hline $\mathrm{f}$ & 80 & $\begin{array}{c}32 \\
(80 \%)\end{array}$ & - & $\begin{array}{c}33 \\
(83 \%)\end{array}$ & - & $\begin{array}{l}4 \mathrm{D} \rightarrow ? \\
4 \mathrm{R} \rightarrow \mathrm{D}, 1 \mathrm{R} \rightarrow \mathrm{F}, \\
3 \mathrm{R} \rightarrow \mathrm{P}, 1 \mathrm{~F} \rightarrow \mathrm{R}, \\
4 \mathrm{~F} \rightarrow \mathrm{D}, 1 \mathrm{~F} \rightarrow \mathrm{P} \\
1 \mathrm{~F} \rightarrow ?\end{array}$ \\
\hline $\mathrm{p}$ & 40 & - & - & - & $\begin{array}{c}33 \\
(83 \%)\end{array}$ & $3 \mathrm{P} \rightarrow \mathrm{D}, 4 \mathrm{P} \rightarrow ?$ \\
\hline otal & 365 & $\begin{array}{c}113 \\
(81 \%)\end{array}$ & $\begin{array}{c}125 \\
(86 \%)\end{array}$ & $\begin{array}{c}33 \\
(83 \%)\end{array}$ & $\begin{array}{c}33 \\
(83 \%)\end{array}$ & 61 \\
\hline
\end{tabular}

aPlates $r, d, f$ and $p$, subject to flaws of type $R, D, F$ and P. The network was trained with 55 spectra representing all flaw types. The percentages given are match scores, referring to the actual occurrence of flawed plate points of specified type.

\section{Further Tests}

The influence of the normalization constant of power spectra was examined. Removing it from the network input did not cause any significant change in the performance of the net. This indicates that the discriminating factor of primary importance is the overall shape of the signal, not its absolute size.

A test at higher resolution was performed on plate d, using two $5 \mathrm{MHz}, 30$ degree miniature angle-beam transducers $5.5 \mathrm{~mm}$ apart. Plate $\mathrm{d}$ was scanned at $5 \mathrm{~mm}$ intervals along a line passing through three delaminated regions. Training examples consisted of six R-spectra from plate $r$ and seven $\mathrm{D}$-spectra from one of the D-regions of plate d. After training, the network classified all except two of the 53 spectra of the scanned line correctly. Borders separating flawed and unflawed material were thus determined within $5 \mathrm{~mm}$.

\section{DISCUSSION AND CONCLUSIONS}

A new concept of flaw detection, based on ANNs, has been proposed for composite materials. As for SWF analysis, the concept relies on the assumption that microstructural material properties affect the transmission of properly induced stress waves and that this effect is reflected in ultrasonic power spectra. In contrast to SWF analysis, the ANN proposal does not rely on explicitly stated rules concerning how changes in spectral properties should be related to changes in material. Instead, such rules evolve implicitly in an ANN through the presentation of many example spectra from specimens subject to known flaws.

The strength of the concept lies in this circumvention of two difficult and critical steps of traditional analysis: the choice of a set of discriminating spectral factors and the determination of a relationship between factor values and flaw state. The weakness of the concept is that considerable amounts of training data are necessary and that it is not possible to gain any insight into the rules by which the network classifies data.

The concept most readily lends itself to applications where many more-or-less identical specimens, subject to a finite number of frequently occurring flaws, are to be checked. Such applications will profit also from the extremely fast performance of trained neural networks, especially when implemented in parallel hardware, and from the possibility of fully automating the flaw-detection process by letting network output neurons correspond directly with the sorting machinery.

The example application presented in this paper, although limited in scope, provides a first indicator of the results to be expected. Much remains to be done with respect to optimization of the measurement setup, the pre-network signal conditioning, and the network itself, as also with respect to performance verification through more comprehensive and realistic tests. These aspects are currently being investigated, together with the possibility of doing graded flaw detection and of correlating power spectra with quantitative physical measures, e.g., elastic moduli.

\section{Acknowledgment}

The authors would like to thank Ramesh Talreja of the Technical University of Denmark for valuable help and stimulating discussions.

\section{References}

1. Talreja, R., "Applications of Acousto-Ultrasonics to Quality Control and Damage Assessment of Composites," in Duke, J. C., ed., Acousto-Ultrasonics, Theory and Application, 1988, pp 177-190. Plenum Press, New York, NY.

2. Vary, A., "Correlations among Ultrasonic Propagation Factors and Fracture Toughness Properties of Metallic Materials," NASA Technical Memorandum NASA TM X-71889, Mar. 1976.

3. Vary, A., and K. J. Bowles, "Use of an Ultrasonic-Acoustic Technique for Nondestructive Evaluation of Fiber Composite Strength," NASA Technical Memorandum NASA TM-73813, Feb. 1978.

4. Rumelhart, D. E., and J. L. McClelland, Parallel Distributed Processing, Explorations in the Microstructure of Cognition, Vol. 1, Foundations, 1986. MIT Press, London, UK.

5. Lippmann, R. P., "An Introduction to Computing with Neural Nets,". IEEE ASSP Magazine, Apr. 1987, pp 4-22.

6. Baker, A. R., and C. G. Windsor, "The Classification of Defects from Ultrasonic Data Using Neural Networks: The Hopfield Method," NDT International, Vol. 22, No. 2, Apr. 1989, pp 97-105.

7. Rumelhart, D. E., G. E. Hinton, and R. J. Williams, "Learning Internal Representations by Error Propagation," in D. E. Rumelhart and J. L. McClelland, eds., Parallel Distributed Processing, Vol. 1, 1986, pp 318-362. MIT Press, London, UK.

\section{Authors}

Jon Juel Thomsen is associate professor at the Technical University of Denmark, Dept. of Solid Mechanics, where he received his M.S.E. in 1985 and Ph.D. in 1988. His research interests include artificial neural networks, biomechanics, adaptive structures, experimental mechanics, and nonlinear vibrations.

Kaspar Lund is company manager at Perceptronics International, Toulouse, France. He received his M.S.E. in 1986 at the Technical University of Denmark, Dept. of Solid Mechanics. He has worked with signal processing, robotics, and software development methods in the companies Matra, S.A., France, and Chr. F. Rovsing A/S, Denmark. He is now working with industrial realization and research of artificial neural networks and signal processing for nondestructive control of materials. 


\section{NATIONAL AERONAUTICS AND SPACE ADMINISTRATION \\ Lewis Research Center \\ Cleveland, Ohio 44135}

October 16,1991

Professor Jon Juel Thomsen

Solid State Mechanics Department

Technical University of Denmark

DK-2800, Lyngby, DENMARK

\section{Dear Professor Thomsen:}

I wish to express my appreciation for the fine article that you and your colleague Kaspar Lund published in the special May 1991 issue of Materials Evaluation on acousto-ultrasonics entitled "Quality Control of Composite Materials by Neural Network Analysis of U1trasonic Power Spectra." It laid down needed foundations for analyzing the complex signal patterns associated with acousto-ultrasonic stress waves. I hope that you are able to continue this work with more extensive training sets comprised of appropriate bench mark samples.

I have enclosed a call for papers for the Second International Conference on AcoustoUltrasonics to be held in Atlanta, Georgia during June 24 and 25, 1993. The Conference is being sponsored by ASNT and ASTM. I look forward to meeting you in the near future, perhaps at the Conference.

Sincerely,

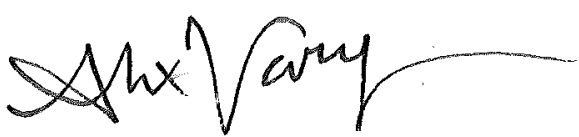

Alex Vary, Deputy Manager

Structural Integrity Branch

Enclosure

cc: Kaspar Lund 